\begin{tabular}{c|c|c|c|} 
SELECCIONES MATEMÁTICAS \\
Universidad Nacional de Trujillo \\
ISSN: $2411-1783$ (Online) \\
2021; Vol. 8(2): 386-403.
\end{tabular}

MATHEMATICS EDUCATION

\title{
Some historical-analytical aspects of functional analysis
}

\author{
Algunos aspectos históricos-analíticos del análisis funcional \\ Alejandro Ortiz Fernández ${ }^{(1)}$ \\ Con afecto a mi profesor Djairo G. de Figueiredo, por su amistad y enseñanzas
}

Received, Oct. 22, 2021

Accepted, Dec. 05, 2021

How to cite this article:

Ortíz A. Some Historical-Analytical Aspects of Functional Analysis. Selecciones Matemáticas. 2021;8(2):386-403. http://dx.doi.org/10.17268/sel.mat.2021.02.15

\begin{abstract}
In ten sections we present an overview of the evolution of functional analysis, from the calculus of variations to partial differential operators, through integral equations and the contributions made in the first half of the 20th century.
\end{abstract}

Keywords . Calculus of variations, Dirichlet principle, operators, Hilbert spaces, distributions.

\section{Resumen}

En diez secciones presentamos un panorama de la evolución del análisis funcional, desde el cálculo de variaciones hasta los operadores diferenciales parciales, pasando por las ecuaciones integrales y por los aportes hechos en la primera mitad del siglo XX.

Palabras clave. Cálculo de variaciones, principio de Dirichlet, operadores, espacios de Hilbert, distribuciones.

1. Panorama General. El análisis funcional se formalizó en la primera mitad del siglo XX pero fue la consolidación de varios métodos e ideas venidas de los dos anteriores siglos. Se trató de entender desde un punto de vista uniforme y general un abultado material que existía en distintas ramas de la matemática, como fueron el cálculo de variaciones, la teoría de ecuaciones integrales, las ecuaciones diferenciales ordinarias y parciales, la teoría de funciones de una variable real; en la teoría de operadores y de la aproximación. En realidad, algunas ideas vinieron del siglo XVIII (ver [10, 11]) cuando investigaciones en la física-matemática y en la mecánica celeste retaron a los matemáticos a introducir nuevas ideas y métodos.

A inicios del sigo XIX la matemática entra en una etapa de rigor y madurez aún cuando existían varias ideas difusas y gaseosas, como hubo en el famoso trabajo de Fourier, pero esas ideas funcionaban bien, como en el caso de la condución del calor. Para corregir tales deficiencias tuvimos el aporte de notables matemáticos como Augustin Cauchy (1789-1857), quien contribuyó a la formulación del análisis matemático; él riguriza el concepto de límite, clarifica la idea de continuidad y considera integrales de funciones con algunos puntos de discontinuidad, ...; le da al análisis un contexto sólido y analítico. Cauchy investiga a las series, clasificándolas en convergentes y divergentes; considera el valor principal de una integral; su obra es una importante contribución a la abstracción de la matemática.

Otro gran matemático que contribuyó a la rigurización del análisis fue Karl F. Gauss (1777-1855) quién investigó con mucha profundidad problemas de la matemática pura y de la matemática aplicada. A él le debemos el uso correcto del paso al infinito pues antes se hacía intuitivamente y con cierto temor. En general remarcamos que a inicios del siglo XIX los matemáticos comenzaron a tener interés por precisar las ideas y

* Sección Matemática, Pontificia Universidad Católica del Perú, Lima, Perú. (jorti z apucp . edu • pe). 
por probar con rigor las aseveraciones. La falta de rigor condujo a paradojas, como fue con las series pues se desconocía los criterios de convergencia. Todo esto generó malestar en el ambiente matemático y debemos las contribuciones de científicos como Cauchy, Gauss y otros para poner orden. Así tenemos a Niels Abel (1802-1829) quién demostró la imposibilidad de resolver algebraicamente toda ecuación de grado superior a cuatro. En el campo del análisis contribuyó en la teoría de la convergencia de series; investigó sobre las integrales abeliana, sobre las funciones elípticas. Abel en una carta de 1826 se queja de "la tremenda oscuridad que encuentra uno en el análisis". Dice aún: "Hay muy pocos teoremas en el análisis superior que se hayan demostrado de una manera lógicamente sostenible, ...” (ver [7], pag. 1250).

¿Qué idea de la derivada se tenía a inicios del siglo XIX?. Newton y Leibniz, tenían una idea clara de la derivada al considerar que ella debe considerarse como el límite de la razón de las diferencias de las variables dependientes y las variables independientes, tal como actualmente la comprendemos. La noción de derivada como razón de cambio fue fundamental en las investigaciones hechas en el siglo XVIII, sobre todo en la física-matemática. Así, por ejemplo, en 1747 d'Alambert estudió el problema de las pequeñas vibraciones de una cuerda de longitud $L$, fija en sus extremos; la cuerda se mueve en un plano cartesiano adecuado. Sea $y=u(x, t)$ la ecuación de la cuerda en el tiempo $t, 0 \leq x \leq L$. d'Alambert probó que bajo ciertas condiciones $u(x, t)$ satisface la ecuación

$$
\frac{\partial^{2} u}{\partial t^{2}}=c^{2} \frac{\partial^{2} u}{\partial x^{2}}
$$

donde $c$ es una constante si la densidad de la cuerda es constante. Además prueba que la solución de tal ecuación es dada por

$$
u(x, t)=f(x-c t)+g(x+c t),
$$

donde $f$ y $g$ son funciones arbitrarias.

La polémica que surgió fue ¿qué se entendía por funciones arbitrarias?. Los matemáticos del siglo XVIII entendían que una función debería tener la misma expresión analítica en todas partes. Euler y Lagrange, a finales del citado siglo consideraron que las funciones pudieran tener diferentes expresiones en dominios diferentes; y la función era continua donde ella mantenía la misma expresión, y era discontinua en puntos donde cambiaba la expresión, cambiaba de forma como se puede ver en la figura 1.1:

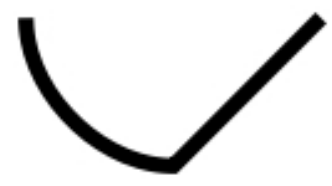

Figura 1.1: Gráfico de una función discontinua

De esta manera el siglo XIX se inicia con una idea no clara, rigurosa, de función.

Le debemos a Bolzano, Abel, Cauchy, Dirichlet, Riemann y Gauss, entre otros matemáticos, la rigurización del cálculo infinitesimal. Posteriormente, Weierstrass hizo contribuciones fundamentales al mejorar las ideas de sus antecesores, como definir correctamente cuando una función $f(x)$ es continua en $x=a$. En 1842 Weierstrass ya tenía la idea de convergencia uniforme, la que ayudaríaa clarificar algunas situaciones. Así, la usó para dar condiciones para integrar término a término en una serie, así como derivar bajo el signo de integral.

En el siglo XIX la matemática progresó mucho, en particular el análisis matemático era cada vez mas riguroso; también comenzó a usarse transformaciones u operadores que actuaban sobre funciones, tal como sucede con los operadores diferenciales, ordinarias y parciales. Otra área que se investigaba, y que también motivó la creación del análisis funcional, fue el cálculo de variaciones, donde se busca maximizar ó minimizar ciertos operadores integrales. En este ambiente surge una teoría abstracta de funcionales!.

2. El Cálculo de Variaciones. Retrocedamos al siglo XVIII. Newton muere en 1727 y el cálculo infinitesimal progresa en diversas ramas; por ejemplo surgen las ecuaciones diferenciales, se estudian algunos problemas de la física-matemática y dentro de este panorama surge una rama nueva, el cálculo de variaciones, que investiga la minimización (ó maximización) de integrales de ciertas funciones y su derivada. En esta dirección el primer problema y su solución fue hecho por Newton (ver [7], pag. 759). 
- Problema: [Newton. Libro II. "Principia”]

"Encontrar el valor mínimo de la integral

$$
J=\int_{x_{1}}^{x_{2}} \frac{y(x)\left[y^{\prime}(x)\right]^{3}}{1+\left[y^{\prime}(x)\right]^{2}} d x
$$

escogiendo la función adecuada $y(x)$, cuyo gráfico rota alrededor del eje $x "$.

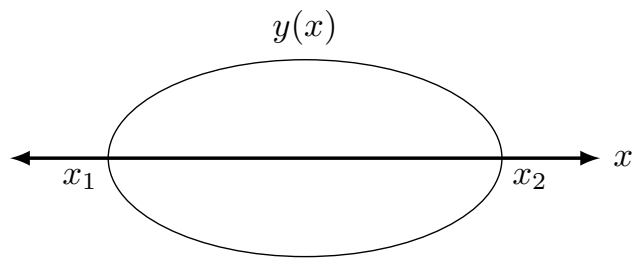

Figura 1: Función $y(x)$ que al girar engendra una superficie con menor área.

Newton llegó a estudiar este problema motivado por el movimiento de los objetos en el agua. Investiga también sobre "la forma que debe tener la superficie de revolución que se mueve a una velocidad constante en la dirección de su eje, si ella ofrece la menor resistencia al movimiento". Así surge el problema de encontrar superficie de revolución de área mínima: "entre las curvas que unen dos puntos de un plano, hallar aquella curva cuyo arco, al rotar alrededor del eje x, engendra la superficie con menor área."

Veamos a donde nos conduce este problema. Sean $P_{1}=\left(x_{1}, y_{1}\right)$ y $P_{2}=\left(x_{2}, y_{2}\right)$ los puntos dados; $y=f(x)$ es la función que describe la curva arbitraria dada satisfaciendo la condición $y_{1}=f\left(x_{1}\right)$ y $y_{2}=f\left(x_{2}\right) .{ }^{(*)} \mathrm{Al}$ girar la curva alrededor del eje $x$, ella describe una superficie cuya área es dada por la integral

$$
J(y)=2 \pi \int_{x_{1}}^{x_{2}} y \sqrt{1+\left(y^{\prime}\right)^{2}} d x .
$$

Problema: Determinar la curva $y=f(x)$, satisfaciendo la condición $(*)$, tal que $J(y)$ asuma un valor mínimo.

Por otro lado, en 1696, John Bernoulli propuso un problema isoperimétrico (Solución de problemas de máximos y mínimos por métodos puramente geométricos) llamado el problema de la braquistócrona ó de la curva de descenso más rápido: "entre todas las curvas que unen los puntos $P_{1}$ y $P_{2}$, se desea hallar la curva que a lo largo de ella una partícula, bajo la fuerza de gravedad, de descenso más rápido desde $P_{1}$ (sin velocidad inicial) llega al punto $P_{2}$ en el menor tiempo."

Veamos algunas ideas al respecto. Sea $P_{1}=(0,0), P_{2}=\left(x_{2}, y_{2}\right)$ y $y=f(x), 0 \leq x \leq x_{2}$, describe una curva arbitraria donde se asume que $f$ es continuamente diferenciable. Se tiene $f(0)=0$ y $f\left(x_{2}\right)=y_{2}$. Vía argumentos físicos, ver por ejemplo [8], pag. 33, se llega a establecer

$$
J(y)=\frac{1}{\sqrt{2 g}} \int_{0}^{x_{2}} \sqrt{\frac{1+\left(y^{\prime}\right)^{2}}{y}} d x,
$$

donde $g$ es la constante de gravedad.

Problema: Entre todas tales posibles curvas, hallar aquella que haga $J(y)$ un mínimo.

Estos problemas, entre otros, son considerados los precursores del análisis funcional. Se trata de maximizar o minimizar expresiones del tipo

$$
J(y)=\int_{a}^{b} F\left(y(x), y^{\prime}(x), \ldots\right) d x
$$

donde $F$ es una función regular y las $y(x)$ s son curvas regulares en el intervalo $[a, b]$. Por otro lado, debemos tener cuidado pues en ciertas condiciones no existe tal curva $y(x)$. En esta historia están relacionados los nombres de Riemann y Weiestrass. En el contexto histórico, si bien el trabajo de John Bernoulli es considerado el origen del cálculo de variaciones, en la lejana Grecia alrededor del año 150 a.C. el matemático 
Zenodorus escribió sobre las figuras isoperimétricas, trabajo que fue difundido por Pappus; se probó, por ejemplo, "que el círculo tiene la mayor área que cualquier polígono regular con el mismo perímetro".

Respecto al problema de la braquistócrona los hermanos Jean y Jacques Bernoulli encontraron en 1697 que la cicloide es la curva solución.

Nota 1. Newton, Leibniz, L'Hopital, John y James Bernoulli también encontraron la solución correcta del problema de la braquistócroma, cuyas soluciones fueron publicadas en el Acta Eruditorum, mayo 1697.

2.1. Las Ecuaciones Diferenciales del Cálculo de Variaciones de Euler. En 1728 Euler, motivado por un problema propuesto por Jean Bernoulli, se inicia en el cálculo de variaciones en donde hace notables contribuciones. Veamos; sea la funcional $J(x)=\int_{t_{1}}^{t_{2}} F\left(t, x, x^{\prime}\right) d t$; Euler observa que la función $F$ satisface la ecuación diferencial ordinaria $\frac{d}{d t} F_{x^{\prime}}-F_{x}=0$. Luego, si la función $x(t)$ minimiza a $J(x)$, ella debe satisfacer a esta ecuación diferencial. Observamos que vía esta ecuación diferencial de Euler se prueba que la solución del problema de la braquistócroma es la cicloide. Por otro lado, los filósofos y científicos afirmaron que "la naturaleza actúa en el camino más corto posible"; así, en el siglo XVII Fermat estableció el Principio del Menor Tiempo: "la luz siempre toma la trayectoria que requiere el menor tiempo".

Euler y Lagrange fundaron el cálculo de variaciones en el siglo XVIII en relación con problemas de la física siendo el principio de la menor acción una de las motivaciones para investigaciones en la física matemática, trabajo que se continuó en el siglo XIX. Veamos algunas ideas matemáticas hacia las ecuaciones diferenciales de Euler. Sea $D$ un conjunto arbitrario y la función $J: D \rightarrow \mathbb{R}$. Asumamos que existe una constante $m$ (ó $M$ ) tal que $m \leq J(x)$ (ó $J(x) \leq M), \forall x \in D$.

Pregunta: ¿Existe $x_{0} \in D$ tal que $J\left(x_{0}\right)=m\left(\right.$ ó $\left.J\left(x_{0}\right)=M\right)$ ?

Veamos. Si $D=[0,1]$ y $J=f$ es continua en D, entonces la respuesta es afirmativa. Si $D$ fuera un espacio topológico compacto y $J=f$ es continua, la respuesta es también afirmativa. Si $D$ fuera un espacio de funciones, $J: D \rightarrow \mathbb{R}$ es llamada una funcional. En este caso la respuesta puede ser falsa. Por ejemplo, sea $D=\{x:[0,1] \rightarrow \mathbb{R}$ continua $/ x(0)=1=x(1)\}$ y sea la funcional $J(x)=\int_{0}^{1} x^{2}(t) d t$. Para afirmar que ínf $J(x)=0$, observar el siguiente gráfico. Pero no existe $x_{0} \in D$ tal que $J\left(x_{0}\right)=0$.

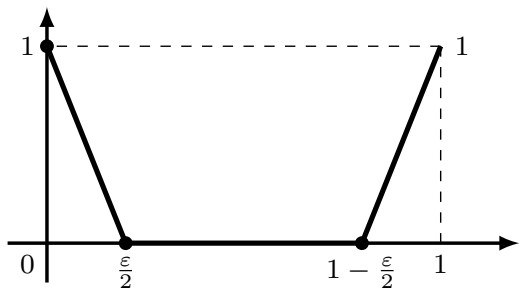

Figura 2: Contraejemplo a un problema de minimización.

Ver [4], pag. 21, para otros comentarios.

Definición 1. Un problema variacional consiste en hallar una función en $D$ que minimice o maximice a la funcional $J$.

Ahora nos dirigimos a las ecuaciones de Euler; para ello consideramos dos ejemplos:

(i) Sea

$$
D=\left\{x:[a, b] \rightarrow \mathbb{R}, x \in C^{2}([a, b]) / x(a)=x_{1}, x(b)=x_{2}\right\},
$$

y la funcional $J(x)=\int_{a}^{b} F\left(t, x, x^{\prime}\right) d t$, donde $F \in C^{2}\left(t, x, x^{\prime}\right)$.

Hallar $x_{0}(t) \in D$ tal que minimice a $J(x)$.

(ii) (Extensión de (i)). Sea

$$
D=\left\{(x(t), y(t)), x, y \in C^{2}([a, b]) / x(a)=x_{1}, y(a)=y_{1}, x(b)=x_{2}, y(b)=y_{2}\right\},
$$


y la funcional $J(x, y)=\int_{a}^{b} F\left(t, x, y, x^{\prime}, y^{\prime}\right) d t$, donde $F \in C^{2}\left(t, x, y, x^{\prime}, y^{\prime}\right)$.

Hallar $\left(x_{0}(t), y_{0}(t)\right) \in D$ tal que minimice a $J(x, y)$.

Teorema 1. Sea $x_{0}=\varphi(t)$ e $y_{0}=\psi(t)$ solución del problema (ii), entonces ella es también solución del sistema de ecuaciones diferenciales:

$$
\left\{\begin{array}{l}
\frac{d}{d t} F_{x^{\prime}}-F_{x}=0, \\
\frac{d}{d t} F_{y^{\prime}}-F_{y}=0 .
\end{array}\right.
$$

(Ecuaciones de Euler)

Demostración: Si $J(x, y)=\int_{a}^{b} F\left(t, x, y, x^{\prime}, y^{\prime}\right) d t$ asume su mínimo en $(\varphi, \psi)$ se tiene

$$
J(\varphi, \psi) \leq J\left(\varphi+\varepsilon_{1} \eta_{1}, \psi+\varepsilon_{2} \eta_{2}\right), \forall \varepsilon_{1}, \varepsilon_{2}>0,
$$

y $\eta_{1}=\eta_{1}(t), \eta_{2}=\eta_{2}(t)$ son funciones de clase $C^{2}([a, b])$ que se anulan en $a$ y $b$. Ahora definimos la función de valor real en las variables $\varepsilon_{1} \mathrm{y} \varepsilon_{2}$ :

$$
q\left(\varepsilon_{1}, \varepsilon_{2}\right)=\int_{a}^{b} F\left(t, \varphi+\varepsilon_{1} \eta_{1}, \psi+\varepsilon_{2} \eta_{2}, \varphi^{\prime}+\varepsilon_{1} \eta_{1}^{\prime}, \psi^{\prime}+\varepsilon_{2} \eta_{2}^{\prime}\right) d t
$$

la cual asumimos toma su mínimo en el origen, lo que implica que las primeras derivadas son nulas en $(0,0)$. Así tenemos (pensando en integración por partes):

$$
0=\int_{a}^{b} \eta_{1} F_{x} d t+\int_{a}^{b} \eta_{1}^{\prime} F_{x^{\prime}} d t=\int_{a}^{b} \eta_{1} F_{x} d t+\left.\eta_{1} F_{x^{\prime}}\right|_{a} ^{b}-\int_{a}^{b} \eta_{1} \frac{d}{d t} F_{x^{\prime}} d t=\int_{a}^{b}\left(F_{x}-\frac{d}{d t} F_{x^{\prime}}\right) \eta_{1} d t
$$

En forma similar se obtiene

$$
\int_{a}^{b}\left(F_{y}-\frac{d}{d t} F_{y^{\prime}}\right) \eta_{2} d t=0 .
$$

Ahora usamos el lema fundamental del Cálculo de variaciones:

"Sea $f: D \subset \mathbb{R}^{n} \rightarrow \mathbb{R}$ una función continua. Si para toda función real continua $\eta(x)$ sobre $D$, tal que $\eta(x)$ es nula en una vecindad del controno de $D$ se tuviera $\int f \cdot \eta d x=0$, entonces $f(x)=0, \forall x \in D^{\prime}$

Vía este lema tenemos finalmente: $\frac{d}{d t} F_{x^{\prime}}-F_{x}=0 \mathrm{y} \frac{d}{d t} F_{y^{\prime}}-F_{y}=0$.

Con el objetivo de obtener la ecuación de Euler para funciones que dependen de más de una variable (por simplicidad $n=2$ ) veamos el siguiente ejemplo:

(iii) Sea $V \subset \mathbb{R}^{2}$ un dominio regular (con contorno $\partial V$ regular) y

$$
D=\left\{u=u(x, y): V \rightarrow \mathbb{R} \text { de clase } C^{2}(V) / u=f \text { sobre } \partial V\right\} .
$$

Sea la funcional $J(u)=\int_{V} F\left(x, y, u, u_{x}, u_{y}\right) d x d y$, donde $u_{x}=\frac{\partial u}{\partial x}, F \in C^{2}\left(x, y, u, u_{x}, u_{y}\right)$. Considere el respectivo problema variacional.

Teorema 2. Sea $u_{0}=\varphi(x, y)$ una solución del problema variacional (iii), entonces $\varphi(x, y)$ es solución de la ecuación diferencial parcial

$$
\frac{\partial}{\partial x} F_{u_{x}}+\frac{\partial}{\partial y} F_{u_{y}}-F_{u}=0
$$

(Ecuación de Euler)

Demostración: Asumamos que $J(u)$ asume su mínimo en $u_{0}=\varphi(x, y)$ en $D$. Luego

$$
J(\varphi) \leq J(\varphi+\varepsilon \eta), \forall \varepsilon>0, \eta \in C^{2}(V),
$$

con $\eta=0$ sobre $\partial V$. Ahora se define

$$
q(\varepsilon)=\int_{V} F\left(x, y, \varphi+\varepsilon \eta, \varphi_{x}+\varepsilon \eta_{x}, \varphi_{y}+\varepsilon \eta_{y}\right) d x d y
$$


$q(\varepsilon)$ toma su valor mínimo en $\varepsilon=0$, luego se debe tener $q^{\prime}(0)=0$, de donde (argumento del Teorema 1 )

$$
\int_{V}\left[\eta F_{u}\left(x, y, \varphi, \varphi_{x}, \varphi_{y}\right)+\eta_{x} F_{u_{x}}\left(x, y, \varphi, \varphi_{x}, \varphi_{y}\right)+\eta_{y} F_{u_{y}}\left(x, y, \varphi, \varphi_{x}, \varphi_{y}\right)\right] d x d y=0
$$

Usando $\eta=0$ sobre $\partial V$ se tiene

$$
\int_{V}\left(F_{u}-\frac{\partial}{\partial x} F_{u_{x}}-\frac{\partial}{\partial y} F_{u_{y}}\right) \eta d x d y=0
$$

y usando el lema fundamental se obtiene la tesis.

Nota 2. A inicios del siglo XVIII los matemáticos eran cada vez más concientes de que la naturaleza procura maximizar ó minimizar la realidad del mundo físico. Euler postuló que los fenómenos naturales buscan optimizar la realidad física.

2.2. El Principio de Dirichlet. El cálculo de variaciones tuvo diversas aplicaciones en la geometría y en la física; la teoría estuvo relacionada con un notable problema del siglo XIX: el problema de Dirichlet (ver [4] y [8]), el cual consiste en "encontrar una función armónica $u(x, y)$ en un dominio $\Omega \subset \mathbb{R}^{2}$ (por simplicidad) tal que $u=f$ sobre el contorno $\partial \Omega$, siendo $f$ una función continua dada sobre $\partial \Omega$ ”. La solución del problema de Dirichlet, si existe, es única; además, ella depende continuamente de los datos de contorno. Remarcamos que $u(x)$ es armónica en $\Omega \subset \mathbb{R}^{n}$ si $\Delta u(x)=0$, donde $\Delta=\sum_{i=1}^{n} \frac{\partial^{2}}{\partial x_{i}^{2}}$.

$$
\left\{\begin{array}{l}
\text { El problema variacional de minimizar la funcional }\left(\text { en } \mathbb{R}^{2}\right) J(u)=\int_{V}\left(u_{x}^{2}+u_{y}^{2}\right) d x d y \\
\text { (integral de Dirichlet) en el dominio } D(f)=\left\{u \in C^{2}(V) / u=f \text { sobre } \partial V\right\}
\end{array}\right.
$$

tiene por ecuación de Euler $\Delta u \equiv u_{x x}+u_{y y}=0$. En efecto, tenemos $F\left(x, y, u, u_{x}, u_{y}\right)=u_{x}^{2}+u_{y}^{2}$, luego $F_{u_{x}}=2 u_{x}, F_{u_{y}}=2 u_{y}, F_{u}=0$. Entonces la ecuación de Euler es $2 u_{x x}+2 u_{y y}=0$ ó $\Delta u=0$ con $u=f$ sobre $\partial V$.

Por lo tanto: Si el citado problema variacional tiene solución $u$, ella es también solución del problema de Dirichlet:

$$
\left\{\begin{aligned}
u_{x x}+u_{y y}=0 & & \text { en } V \\
u=f & & \text { sobre } \partial V
\end{aligned}\right.
$$

El recíproco de la anterior aseveración es también cierta. En efecto, si $u$ solución del citado problema de Dirichlet, entonces $u$ es solución del mencionado problema variacional.

Probemos esto. Si $v$ fuera otra solución del problema de Dirichlet citado en $D(f)$, sea $w=v-u$ ó $v=$ $u+w$, con $w=f-f=0$ sobre $\partial V$. Luego,

$$
\begin{aligned}
J(v) & =J(u)+J(w)+2 \int_{V}\left(u_{x} w_{x}+u_{y} w_{y}\right) d x d y \\
& =J(u)+J(w)+2 \int_{V}\left(u_{x x}+u_{y y}\right) w d x d y \\
& =J(u)+J(w) \geq J(u) .
\end{aligned}
$$

Luego $J(u)$ es mínimo en $D(f)$.

Teorema 3 (Principio de Dirichlet). "Una solución del problema variacional $(P V)$ coincide con una solución del problema de Dirichlet $(P D)$ ".

Riemann, en 1851, atacó el problema de Dirichlet usando argumentos de minimizar a la citada funcional $J(u)$ y supuso que existe el minímo de $J(u)$ lo cual justificó por razones físicas. A esta suposición Riemann llamó "Principio de Dirichlet". Este trabajo mereció la crítica de Weierstrass, quien en 1870 observó que el método usado por Riemann era incompleto pues no prueba que existe una función que minimice a $J(u)$ (ver la excelente monografía de D. Figueiredo [4], Cap. V). Por otro lado, debemos observar que los teoremas que garantizan la existencia del mínimo (máximo) de una funcional dependen de la idea de compacidad y así vamos entrando en el análisis moderno. D. Hilbert, a inicios del siglo XX, clarifica al Principio de Dirichlet. 
3. Las Ecuaciones Integrales. Según Dieudonné, [3], un trabajo de Ivar Fredholm de 1900 sobre ecuaciones integrales fue fundamental para el desarrollo del análisis funcional a inicios del siglo XX. (El nombre de ecuación integral fue usado por primera vez por P. du Bois-Reymond en 1888 cuando trabajaba sobre el problema de Dirichlet). El desarrollo de estas ecuaciones fue influenciado por diversos problemas surgidos en la física y así con las ecuaciones diferenciales. Así, el método de Neumann para resolver el problema de Dirichlet conduce a una ecuación integral de la forma $f(x)=u(x)+\int_{a}^{b} K(x, y) u(y) d y$, donde $u(x)$ es la función incógnita y $f(X), K(x, y)$ son funciones conocidas. Esta es una ecuación integral de segunda especie dado que la incónita está dentro y fuera de la integral. Por otro lado, Abel en 1823 al estudiar el problema de la tautócrona resuelve la ecuación $f(x)=\int_{0}^{x} \frac{u(y)}{\sqrt{x-y}} d y, x>0$, donde $f(x)$ es conocida y $u(x)$ es la incógnita. Esta ecuación es de primera especie pues la incógnita $u(x)$ solo aparece dentro de la integral. En general Abel estudió ecuaciones integrales de primera especie de la forma $f(x)=\int_{a}^{x} K(x, y) u(y) d y$, mientras que Lieuville trabajó con ecuaciones de la forma (segunda especie) $u(x)=f(x)+\int_{a}^{x} K(x, y) u(y) d y . K(x, y)$ es el núcleo de la ecuación integral. Ecuaciones con $x$ en la parte superior de la integral se llaman ecuaciones de Volterra y si $x=b$, un número fijo, se llaman ecuaciones de Fredholm. Si $f(x)=0$ en la ecuación de segunda especie, la ecuación es homogénea.

Las contribuciones de Abel y de Lieuville, entre otros, motivaron el interés por investigar las ecuaciones integrales, sobre todo por su relación con el problema de Dirichlet para la ecuación del potencial $\Delta u=0$. Así, problemas en ecuaciones diferenciales, ordinarias y parciales, se transforman en ecuaciones integrales, impulsando entonces la investigación de éstas, y de alguna manera estimuló el surgimiento del análisis funcional.

Vito Volterra (1860-1940). Matemático, físico e ingeniero italiano comienza a publicar, en 1884, sobre ecuaciones integrales; en 1896-97 introduce un método para resolver una ecuación de segunda especie de la forma

$$
f(x)=u(x)+\int_{a}^{b} K(x, y) u(y) d y
$$

donde $u(x)$ es la incógnita, con la restricción $K(x, y)=0$ para $y>x$.

Para hallar la solución de (E1) (ver [7], pags. 1394-5) Volterra escribe la ecuación (E1) en la forma

$$
f(x)=u(x)+\int_{a}^{x} K(x, y) u(y) d y
$$

y construye la sucesión $\left\{f_{n}(x)\right\}$ donde $f_{1}(x)=-\int_{a}^{b} K(x, y) f(y) d y, f_{2}(x)=-\int_{a}^{b} K(x, y) f_{1}(y) d y, \ldots$, $f_{n}(x)=-\int_{a}^{b} K(x, y) f_{n-1}(y) d y$. Ahora define $u(x)$ vía:

$$
u(x)=f(x)+\sum_{m=1}^{\infty} f_{m}(x)
$$

Volterra prueba que la serie es convergente y que $u(x)$, así definida, es solución de (E1) y tiene la forma

$$
u(x)=f(x)+\int_{a}^{b} \bar{K}(x, y) f(y) d y
$$

donde $\bar{K}(x, y)$ es un núcleo apropiado.

Volterra estudió también ecuaciones integrales de primera especie de la forma $f(x)=\int_{a}^{x} K(x, y) u(y) d y$ la cuales redujo a ecuaciones de segunda especie. Obseva, en 1896, que una ecuación integral de primera especie puede ser interpretado como límite de un sistema de $n$ ecuaciones lineales con $n$ funciones incógnitas, cuando $n \rightarrow \infty$. Además, estableció teoremas de existencia y unicidad para ecuaciones integrales de la forma $f(x)=u(x)+\int_{a}^{x} K(x, y) u(y) d y$, donde el núcleo debe satisfacer ciertas hipótesis. (ver [7], Cap. 45 ; [5] para otros comentarios).

Erik Ivar Fredholm (1866-1927). Matemático, nació en Estocolmo, fue alumno de Mittag-Leffler. Trabajó en las ecuaciones en derivadas parciales, en particular en el problema de Dirichlet destancándose su trabajo de 1900 en donde investiga un nuevo método para resolver el problema, desarrollando la teoría de 
las ecuaciones integrales. Este trabajo ("Sur une nouvelle méthode pour la résolution du problème de Dirichlet") motivó nuevas ideas en la evolución del análisis moderno. Bajo la influencia de Poincaré, en la ecuación integral introduce un parámetro complejo $\lambda$, estudiando así ecuaciones de la forma

$$
f(x)=u(x)+\lambda \int_{a}^{b} K(x, y) u(y) d y,
$$

en función de $\lambda$. En 1903 Fredholm prueba un resultado fundamental:

"Si $f(x)$ es una función continua, la ecuación (+) tiene solución única si y solo si la ecuación homogénea asociada posee como solución a la trivial".

En esta dirección, Fredholm observa que la ecuacióm homogénea $u(x)=\lambda \int_{a}^{b} K(x, y) u(y) d y$ tiene infinitas soluciones las que son llamadas autofunciones. En este escenario, Fredholm establece el llamado la alternativa de Fredholm, resultado que fue establecido posteriormente con el lenguaje de los espacios de Hilbert, Banach, y de la teoría de operadores sobre tales espacios y ... ya estamos en el mundo del análisis funcional. El lector interesado en estas ideas puede consultar M. Kline, [7] pag. 1387; D. Figueiredo, [5], una excelente monografía sobre las ecuaciones integrales.

En esta ruta, digamos algo más. Cuestiones del mundo físico conducen a ecuaciones integrales, así problemas de oscilaciones libres de un sistema elástico llevan a ecuaciones de primera especie, y cuando las oscilaciones están sometidas a fuerzas externas el problema conduce a ecuaciones de segunda especie. Por otro lado, ciertos problemas exigen que el núcleo sea simétrico, esto es $\mathrm{K}(\mathrm{x}, \mathrm{y})=\mathrm{K}(\mathrm{y}, \mathrm{x})$ y en este caso, las autofunciones y los autovalores ( $\lambda$ 's) de la ecuación integral tienen importantes propiedades. Por ejemplo, existe una sucesión de autovalores reales $\lambda_{1}, \lambda_{2}, \ldots, \lambda_{n}, \ldots$, y a cada autovalor le corresponde una o varias autofunciones. Además, si $\lambda_{i} \neq \lambda_{j}$ y $u_{i}, u_{j}$ son sus correspondientes autofunciones, entonces $u_{i} \perp u_{j}$ (son ortogonales). De esta manera, $\left\{u_{i}\right\}$ es, en este caso, un sistema ortogonal.

Las ecuaciones integrales fueron, y son, importantes porque son la primera rama del análisis funcional; ellas motivaron el desarrollo de la teoría general de operadores lineales, la cual clarificó muchas cuestiones del análisis y de la física moderna (la mecánica cuántica). Remarcamos que en una ecuación integral de la forma $f(x)=\int_{a}^{b} K(x, y) u(y) d y$, donde se dá un núcleo $K(x, y)$, ella hace corresponder a cada $u(x)$ otra función $f(x)$. Este tipo de correspondencia se llama un operador. La teoría de operadores lineales tuvo su origen en problemas de contorno para ecuaciones diferenciales ordinarias y parciales. Todo esto es un amplio universo!

4. David Hilbert. Surge el Análisis Funcional. A fines del siglo XIX e inicios del XX se produjeron progresos en la matemática y en la física que conducirían a grandes teorías; en particular en el análisis matemático. Así, el legado del análisis de Fourier contribuyó poderosamente en la creación de revolucionarias teorías. Las ecuaciones en derivadas parciales hicieron progresar a la física teórica; en este escenario destacamos al problema de Dirichlet para la ecuación de Laplace $\Delta u=0$ en donde Riemann consideró al llamado Principio de Dirichlet que consiste en minimizar a la funcional

$$
J(u)=\iint_{V}\left[\left(\frac{\partial u}{\partial x}\right)^{2}+\left(\frac{\partial u}{\partial y}\right)^{2}\right] d x d y
$$

donde $u$ es una función continua definida sobre $V \cup \partial V$ ( $V$ un dominio regular en el plano); $u=f$ sobre $\partial V$. Riemann conjeturó que tal mínimo existe, lo cual fue cuestionado (1870) por Weierstrass vía un contraejemplo. Hubo que esperar hasta 1899 cuando D. Hilbert recupera al Principio imponiendo condiciones sobre $f$ y sobre $\partial V$ para garantizar la existencia de la función que minimiza a $J(u)$ y que resuelve al problema de Dirichlet.

Uno de los métodos para resolver el problema de Dirichlet fue hecho por Carol Newmann, el cual condujo a una ecuación integral de la forma $f(x)=u(x)+\lambda \int_{a}^{b} K(x, y) u(y) d y$, donde $\lambda$ es un parámetro complejo, ecuación que como sabemos fue investigada por Fredholm. Se estableció que esta ecuación integral es el límite de la ecuación algebraica

$$
f_{m}=u_{m}+\lambda \sum_{k=1}^{n} K_{m k} u_{k}
$$


Motivado por las investigaciones de Fredholm, Hilbert se interesa por las ecuaciones integrales y estudia el comportamiento de las soluciones de (4.1) cuando $n \rightarrow \infty$ y verifica que estas soluciones tienen por límite a la solución de la ecuación integral dada arriba. Así, en este escenario de los primeros años del siglo XX, Hilbert va considerando nuevas ideas; él era ya una autoridad internacional; se propone llevar la geometría y el análisis del espacio $\mathbb{R}^{n}$ al espacio límite $\mathbb{R}^{\infty}$ surgiendo los espacios de Hilbert $\ell^{2}$. Así, en el sistema infinito de ecuaciones, $m=1,2,3, \ldots$, (4.1) se asume la condición $\sum_{i=1}^{\infty} u_{i}^{2}<\infty$ para que en la citada ecuación integral las funciones que aparecen ahí sean representables usando sus coeficientes de Fourier. Así vino al mundo el espacio $\ell^{2}$; la integral se convierte en una serie.

Se observó que $\ell^{2}\left(\mathbb{R}^{n}\right)$ es un espacio normado completo; Hilbert y sus discípulos investigaron las semejanzas geométricas del espacio $\mathbb{R}^{n}$ con $\ell^{2}$ que es un espacio de dimensión infinita. Remarcamos que el paso de lo finito a lo infinito, de lo continuo a lo discreto, y la identificación vía isomorfismos fue una tarea en esos años, primeros del siglo XX; fue un movimiento revolucionario en el análisis; en verdad en la física clásica también hubo dificultades para explicar fenómenos que surgían en las radiaciones de ciertos cuerpos (negros). Esto indujo a Max Planck (1858-1947) a postular que la emisión y la absorción de la energía se produce siempre en paquetes, en forma "discontinua”, pero también se tenía la teoría ondulatoria ó "continua"; y estas dos teorías no fueron contradictorias! El análisis funcional fue el lenguaje que ayudó a formular la consistencia de ambas teorías. Por su parte los analistas estaban madurando la creación de espacios abstractos.

París, 1900. Se celebra el II Congreso Internacional de Matemática. Hilbert (1862-1943) ofrece una conferencia y propone 23 seleccionados problemas para ser resueltos en el siglo XX. Hilbert era ya famoso; convirtió a Gotinga en el mayor centro matemático del mundo. De 1904 a 1912 investiga el principio de Dirichlet, al cálculo de variaciones y a las ecuaciones integrales. Pone los fundamentos de lo que sería el análisis funcional; fue un gran Maestro y formó matemáticos que continuaron su obra en el campo del análisis. Así tenemos a Erhard Schmidt (1876-1959) quién continuó con las investigaciones sobre las ecuaciones integrales; exploró la semejanza geométrica del espacio $\ell^{2}$ con el espacio $\mathbb{R}^{n}$. Otro joven matemático fue Friedrich Riesz (1880-1956), notable analista húngaro, quien también aportó a las ecuaciones integrales, las que Hilbert había estudiado, sobre todo aquellas de la forma (E1) donde $f(x), K(x, y)$ son funciones continuas. Riesz trata de extender estas ecuaciones para funciones más generales pero, para ello debe estar seguro que los coeficientes de Fourier de $f$ con respecto a cierto sistema ortonormal de funciones, se podían calcular.

Ante esta situación, Riesz considera funciones cuyos cuadrados son integrables según Lebesgue (espacios $L^{2}$ !) y establece que existe una correspondencia biunívoca entre $L^{2}$ y $\ell^{2}$.Además, usando funciones integrables-Lebesgue, prueba que la ecuación (E1) admite solución si $f(x)$ y $K(x, y)$ están en $L^{2}$ (una condición más débil); la solución es única, salvo una función $f$ tal que $\int_{a}^{b} f=0$. Por otro lado, en este escenario surge el analista Ernst Fischer (1875-1959) quien contribuyó también al surgimiento del análisis funcional; en 1907 introduce la idea de convergencia en media. (La sucesión $\left\{f_{n}(x)\right\}$, definidas sobre $[a, b]$, converge en media si $\operatorname{lím}_{m, n \rightarrow \infty} \int_{a}^{b}\left[f_{n}(x)-f_{m}(x)\right]^{2} d x=0 .\left\{f_{n}(x)\right\}$ converge en media a $f(x)$ si $\lim _{n \rightarrow \infty} \int_{a}^{b}\left[f(x)-f_{n}(x)\right]^{2} d x=0$, donde las integrales son en el sentido de Lebesgue. $f$ es única salvo una función definida sobre un conjunto de medida cero). Fischer, independiente de Riesz, también establece el isomorfismo entre $L^{2}$ y $\ell^{2}$ probando que $L^{2}$ es un espacio completo en media, lo que es una ventaja de trabajar con funciones en $L^{2}[a, b]$.

Ordenando los anteriores comentarios (con lenguaje actual) decimos: "Sea $\left\{f_{i}(x)\right\}, i=1,2, \ldots$, una sucesión ortonormal completa en un espacio de Hilbert $H, f \in H$ y $\alpha_{i}, i=1,2, \ldots$ son sus respectivos coeficientes de Fourier $\left(\alpha_{i}=\left(f_{i}, f\right)\right)$, entonces $\sum_{i=1}^{\infty} \alpha_{i}^{2}<\infty$ y $f(x)=\sum_{i=1}^{\infty} \alpha_{i} f_{i}(x)$ ”.

El recíproco de esta afirmación es el famoso

Teorema de Riesz-Fisher: "Sea $\left\{f_{i}(x)\right\}, i=1,2, \ldots$, un conjunto ortonormal en un espacio de Hilbert $H$ $y\left\{\alpha_{i}\right\}, i=1,2, \ldots$, una sucesión de números complejos en $\ell^{2}$, entonces existe $f \in H$ tal que $\alpha_{i}=\left(f, f_{i}\right)$ (producto interno) y $f(x)=\sum_{i=1}^{\infty} \alpha_{i} f_{i}(x)$ ”.

Nota 3.

(i) Es oportuno remarcar que las integrales consideradas en todo lo comentado anteriormente son integrales en el sentido de la teoría de la medida de H. Lebesgue, una teoría introducida a inicios del siglo $X X$. 
(ii) Se observó que la integral $\int_{a}^{b} K(x, y) u(y) d y$ puede ser interpretada como un operador que transforma $u(x)$ en otra función ó en si misma. Y así las ecuaciones integrales motivaron el surgimiento del análisis funcional.

Por otro lado, los anteriores argumentos condujeron a F. Riesz, en 1910, introducir los espacios $L^{p}, 1<$ $p<\infty$, pues deseaba tener la inecuación

$$
\left|\int_{D} f(x) g(x) d x\right| \leq\left(\int_{D}|f(x)|^{p} d x\right)^{\frac{1}{p}}\left(\int_{D}|q(x)|^{q} d x\right)^{\frac{1}{q}}
$$

donde $\frac{1}{p}+\frac{1}{q}=1$. Así, considera al espacio

$$
L^{p}\left(\mathbb{R}^{n}\right)=\left\{f \text { medibles-Lbesgue } / \int_{\mathbb{R}^{n}}|f(x)|^{p} d x<\infty\right\} .
$$

Similar argumento se tiene para el caso discreto,

$$
\sum_{i=1}^{n}\left|x_{i} y_{i}\right| \leq\left(\sum_{i=1}^{n}\left|x_{i}\right|^{p}\right)^{\frac{1}{p}}\left(\sum_{i=1}^{n}\left|y_{i}\right|^{q}\right)^{\frac{1}{q}}, \frac{1}{p}+\frac{1}{q}=1 .
$$

Para mayores argumentos ver [7], Cap. 45.

El análisis funcional debe mucho a las contribuciones de F. Riesz, quién en 1910 clarificó el panorama de entonces; así, por ejemplo, estableció que $L^{p}$ y $L^{q}, \frac{1}{p}+\frac{1}{q}=1$, son espacios duales. Su excelente libro, un clásico, junto con B. Nagy [13] recoge mucho de sus ideas y del estado del análisis funcional en su época. Respecto a los espacios $L^{1}=\left\{f\right.$ medible $\left./ \int_{\mathbb{R}^{n}}|f(x)| d x<\infty\right\}$ y $L^{\infty}=\left\{f\right.$ medible $\left./ \sup _{x}|f(x)|<\infty\right\}$ se estableció que ellos son también espacios duales; en 1918 Hugo Steinhaus verifica que "toda forma lineal continua sobre $L^{1}(I)$, I un intervalo acotado, es de la forma

$$
g \longrightarrow \int_{I} f(x) g(x) d x, \text { donde } g \in L^{1}(I), f \in L^{\infty}(I) . ”
$$

Riesz, en 1918, escribe un notable trabajo sobre la teoría de Fredholm en donde prueba que el espacio de las funciones continuas sobre un intervalo compacto $I, C(I)$, es un espacio completo. Además, conjetura que la teoría de Fredholm podría ser puesta en la forma más general: "todo espacio normado localmente compacto, es de dimensión finita”.

En este escenario están en pleno desarrollo y aceptación la teoría de conjuntos de Cantor y la teoría de la medida e integral de Lebesgue; además, la introducción de los espacios abstractos vino a contribuir mucho en el desarrollo del análisis moderno y de la matemática en general. Maurice Fréchet en 1906 inició el estudio de estos espacios, en particular introduce los espacios métricos; en esta dirección tenemos las contribuciones de Felix Hausdorff con diversas ideas topológicas. También mencionamos a J. Hadamard quién en 1903 ya usa la noción de dualidad topológica y en 1897 sugirió considerar las curvas como puntos de un conjunto; además, estudiando el cálculo de variaciones es motivado a investigar las funcionales.

5. Inicios de la Teoría de Operadores. Alrededor de fines del siglo XIX la idea general de funcional (sea $X$ un espacio abstracto; la transformación lineal $T: X \rightarrow \mathbb{R}$ es llamada una funcional lineal) había aparecido en las investigaciones del cálculo de variaciones y de las ecuaciones integrales; así, de alguna manera la noción general de operador fue gestándose en tal época. (Sean $X$ e $Y$ dos espacios abstractos; la transformación (lineal) $T: X \rightarrow Y$ es llamada un operador (lineal)). En tal épocas tales ideas carecían de una organización rigurosa; recordemos que en 1903 Hadamard introduce la dualidad topológica buscando las funcionales lineales continuas sobre el espacio $C(I)$, espacio de las funciones numéricas donde $I$ es un intervalo compacto. En 1887 la teoría de las funcionales se inicia con Volterra y surgió el problema importante de la representación de funcionales como operadores integrales. En esta dirección se tiene un teorema de representación (1903). En forma independiente, Fréchet y Riesz (en 1907) obtuvieron la representación de una forma lineal continua $T$ sobre el espacio $L^{2}$, según la forma $T(f)=\int f(x) g(x) d x$, para algún $g \in L^{2}$. Aún, 1909, Riesz prueba que cualquier funcional lineal continua $T$ sobre el espacio $C([a, b])$ se representa como una integral de Stieljes, $T(f)=\int_{a}^{b} f(x) d \alpha(x)$, donde $\alpha(x)$ es una función de variación acotada. (Sea $I=[a, b]$ y $f: I \rightarrow \mathbb{R}$ una función de valor real; para cualquier partición $a_{0}<x_{1}<\ldots<x_{n}=b$ de $I$, sea $V_{\Gamma}=\sum_{i=1}^{n}\left|f\left(x_{i}\right)-f\left(x_{i-1}\right)\right| ; V_{\Gamma} \leq \infty$. Sea $V=\sup _{\Gamma} V_{\Gamma} \cdot f$ es 
llamada de variación acotada si $V<\infty)$.

Como sabemos Riesz, en 1910, introdujo los espacios $L^{p}, 1<p<\infty$; se observó que $L^{q}, \frac{1}{p}+\frac{1}{q}=1$, es identificado con su dual (el dual de $L^{2}$ es el mismo). Se generalizó el teorema de representación para funcionales sobre $L^{p}$, lo que permitió a Riesz asociar a los operadores $T$ sobre $L^{p}$, con adjunto $T^{*}$ sobre $L^{q}$ vía la ecuación $\int_{a}^{b} T[f(x)] g(x) d x=\int_{a}^{b} f(x) T^{*}[g(x)] d x$, donde $f \in L^{p}, g \in L^{q}$. Estas ideas fueron motivadas por el problema: "dado el sistema infinito de ecuaciones $\int f_{i}(x) g(x) d x=e_{i}, i \in I$, donde las funciones $f_{i}$ y escalares $e_{i}$ son dadas, hallar la función $g(x)$ ”. Riesz prueba que esta cuestión es consistente si la solución $g$ está en $L^{q}, \frac{1}{p}+\frac{1}{q}=1$. Además introduce la convergencia débil: $f_{n} \rightarrow f$ débilmente si $\int f_{n}(x) g(x) d x \rightarrow \int f(x) g(x) d x, \forall g \in L^{q}([a, b])$.

Estamos en camino hacia espacios más generales ( $L^{2}$ es un espacio de Hilbert y $L^{p}, 1<p<\infty$, un espacio de Banach) y los operadores actuando sobre ellos.

6. Topología y Espacios Normados. La idea de espacios abstractos se remonta al siglo XVII, a la época del cálculo infinitesimal, cuando se piensa en construir conjuntos cuyos elementos no eran números sino funciones y operar en este universo como se hacía en el cálculo. Esta idea fue evolucionando con aportes de B. Riemann quien en 1854 considera espacios abstractos de dimensión infinita y sugiere la idea de estructura topológica. Así llegamos a inicios del siglo XX. Motivado por sus ideas de topología general, Fréchet contribuyó con la introducción de los espacios abstractos; investiga espacios más generales que el clásico espacio euclideano. En su tesis (1906) introduce los espacios métricos en donde desarrolla sus aspectos topológicos y así formalizar en términos generales los trabajos de Volterra, Cantor y Hadamard. Fréchet estudia las funcionales sobre espacios métricos, la idea de compacidad; nos lega un teorema de representación en $L^{2}$. Introduce también las nociones de filtro, convergencia compacta; introduce unos espacios que generalizan a los espacios de Banach, son localmente convexos y la métrica no surge necesariamente de una norma, Los teoremas fundamentales del análisis funcional se conservan aún en estos espacios, llamados espacios de Fréchet; $C^{\infty}$ es un espacio de Fréchet.

El punto de partida hacia los espacios abstractos, en particular los espacios topológicos, es la noción de espacio vectorial el cual fue introducido por G. Peano al rededor de 1888; en este escenario se generalizó la idea de límite y para ello se necesitó tener una definición de distancia. Esto fue labor de Fréchet y los espacios métricos fueron la puerta de entrada a los topológicos; en este escenario la teoría de conjuntos Cantor y las integrales en el sentido de Lebesgue fueron muy importantes. Se observa que en la topología lo escencial no es la distancia sino la idea de entorno o límite. Fréchet observa que en un espacio topológico, la topología puede venir o no de una distancia, es decir, con la noción de entorno se puede construir una teoría topológica de los espacios abstractos, independiente de la idea de distancia.

Fréchet introdujo muchas ideas como, por ejemplo, la de clausura de un conjunto, punto de acumulación, transformación continua de un conjunto; la de homeomorfismo, ... Fréchet hizo notables contribuciones para el surgimiento de la topología general, tan útil para la elaboración del análisis funcional y otras ramas de la matemática, y aplicaciones.

Por todo lo expuesto, ya estaban las condiciones para la introducción de los espacios normados en donde los trabajos de F. Riesz fueron pioneros. Veamos. En 1894 Stieltjes propueso y resolvió el problema: "hallar una función creciente $g$ definida en $[0, \infty)$ tal que $\int_{0}^{\infty} x^{n} d g(x)=c_{n}(n \geq 0), n=0,1,2, \ldots$ ”. Este problema, llamado problema del momento fue generalizado a la forma $\int_{a}^{b} f_{\alpha}(x) d g(x)=c_{\alpha}$, donde $[a, b]$ es un intervalo compacto en $\mathbb{R}, f_{\alpha}$ son funciones continuas en $[a, b], C_{\alpha}$ son escalares dados; se trata de hallar $g$, una función de variación acotada en $[a, b]$, que satisfaga tal ecuación para $\forall \alpha$. En 1911 Riesz estudió tal sistema de ecuaciones lineales usando sus espacios $L^{p}$; en el período 1916-18 construye la teoría espectral de los espacios de Hilbert y desarrolla la teoría de los operadores compactos; en estos trabajos usa la noción de norma. E. Hally, en 1921, trata por primera vez con espacios normados generales en vez de los conocidos $C([a, b]), \ell^{p}$ y $L^{p}$.

En la evolución del análisis funcional hubieron destacados matemáticos que impulsaron a esta nueva teoría, entre ellos tenemos a F. Riesz, ya mencionado antes. Todas estas contribuciones fueron preparando el terreno que condujo al matemático polaco Stefan Banach (1892-1945) a organizar una teoría general de espacios normados, de funcionales y operadores lineales sobre dichos espacios. Estas ideas las presentó Banach en su tesis doctoral (1922); ahí aparecen los espacios B que después se llamarían espacios de Banach, que marcarían toda una etapa en la historia del análisis. Banach y sus colegas hacen de Polonia el centro 
internacional del análisis funcional. Estamos en 1932. En este año se publican tres libros que habrían de consolidar las conquistas hechas en el análisis funcional; ellos son:

(i) "Théorie des Opérations Linéaires", Stefan Banach.

(ii) "Mathematical Foundations of Quantum Mechanics", John von Neumann.

(iii) "Linear Transformations in Hilbert Space and their Applications to Analysis", Marshall H. Stone.

El libro de Banach fue una valiosa contribución que sirvió de motivación para posteriores progresos en los espacios abstractos; tal obra fue el inicio de una etapa de madurez de los espacios normados; tales progresos condujeron a los espacios vectoriales topológicos, una área bastante general. El libro de Banach contiene sus propias investigaciones así como de otros e inició la investigación de lo que sería las algebras de Banach en la década de los años 30's. Demos algunas ideas matemáticas. Sea $F$ un campo; $A$ es una álgebra sobre $F$ si $A$ es un anillo y un espacio vectorial sobre $F$ y se tiene $\alpha(x y)=(\alpha x) y=x(\alpha y), \forall x, y \in A, \alpha \in F$. (Remarcamos que un anillo $\mathbb{R}$ es un grupo aditivo tal que $f: \mathbb{R} \times \mathbb{R} \rightarrow \mathbb{R}, f(a, b)=a b$ satisfaciendo $(a b) c=a(b c), a(b+c)=a b+a c,(b+c) a=b a+c a)$.

- Una álgebra de Banach, ó una álgebra $B$, sobre el campo de los números complejos, es una álgebra que posee una norma con la cual $B$ es un espacio de Banach (un espacio normado completo) y se satisface $\|x y\| \leq\|x\|\|y\|, \forall x, y \in B$. El campo $\mathbb{C}$ de los números complejos, con norma el valor absoluto, es una álgebra de Banach; también lo es el álgebra $C(X)$ de las funciones continuas sobre $X$ (un espacio compacto, Hausdorff) con la adición y multiplicación puntual, con norma $\|f\|_{\infty}=\sup _{x \in X}|f(x)|$. (En una álgebra de Banach podría existir un elemento identidad $e,\|e\|=1$ tal que $x e=e x=x$ ).

Nota 4. El lector interesado en el libro de Banach debe consultar [1]. (En la biblioteca de CienciasPUCP hay un ejemplar).

7. Álgebras de Banach. Operadores. En las primeras décadas del siglo XX la teoría de operadores interesó a los analistas; como sabemos Hilbert y su Escuela estudiaron a las ecuaciones integrales, a la teoría de Fredholm; fundan la teoría moderna espectral. Así mismo F. Riesz investiga al álgebra de los operadores acotados sobre el espacio de Hilbert $\ell^{2}$ y en 1916 funda su teoría de operadores completamente continuos, también llamados operadores compactos; estudia la correspondiente teoría espectral. Más tarde, en 1923, T. Carleman hizo contribuciones sobre operadores no acotados verificando que algunos resultados de Fredholm y Hilbert son ciertos aún para un tipo más débil de acotación. En este panorama tenemos la investigación de J. von Neumann y de M. H. Stone, quienes en el período 1929-1932 aportaron a la teoría espectral para operadores normales, y en 1929 von Neumann extiende la teoría espectral de operadores hermitianos al caso no-acotado. Remarcamos que la definición general de espacio normado es obra de Banach, H. Hahn y E. Helly en el período 1920-22 y las investigaciones se dirigieron a la teoría de la dualidad y surgen las primeras ideas de una teoría más general generada en el período 1920-30, la teoría de los espacios vectoriales topológicos, los que contienen a los espacios de Hilbert y de Banach, y otros.

Remarcamos que especial atención merecieron las álgebras de Banach Conmutativas, área que fue estudiada por Gelfand y otros analistas. Se introdujo la noción de involución en una álgebra de Banach: $*: B \rightarrow B, x \rightarrow x^{*}$, tal que $(x+y)^{*}=x^{*}+y^{*},(x y)^{*}=y^{*} x^{*},(\lambda x)^{*}=\bar{\lambda} x^{*}, \forall$ escalar $\lambda, \mathrm{y}\left(x^{*}\right)^{*}=x$; además se tiene $\left\|x^{*} x\right\|=\|x\|^{2}$. Una álgebra de Banach con involución se llama $C^{*}$-álgebra (remarcamos que el álgebra es conmutativa). Gelfand-Naimark investigaron estas $C^{*}$-álgebras. Para mayores detalles ver [3], pag. 187.

El análisis funcional se desarrolla intensamente en los años 1930's y años posteriores; se interrelacionan métodos algebraicos y topológicos. Se tienen las contribuciones de von Neumann en la teoría espectral de operadores; se interesa por ciertas subálgebras involutivas provistas de apropiadas propiedades topológicas; todo ello condujo a las llamadas álgebras de von Neumann. Motivado por estos avances, I. Gelfand introduce la teoría general de las álgebras normadas en 1941; en ella extiende la teoría espectral de F. Riesz a álgebras normadas; también introdujo la fórmula del radio espectral; dió una nueva explicación de la teoría espectral de Hilbert, Riesz y de von Neumann para operadores normales sobre un espacio de Hilbert. Para detalles sobre la teoría espectral de Hilbert, en esta dirección, ver [3], pag. 148. Por otro lado, Banach observó que existen espacios vectoriales que no son normables pero sí metrizables lo que motivó pensar que existen otros tipos de espacios; Banach, también, consideró espacios que no son metrizables con ciertas topologías débiles. Todo esto motivó al matemático ruso Andrei Kolmogorov, en 1934, a introducir la 
noción de espacio vectorial topológico (e.v.t), el cual es un espacio vectorial provisto de una topología que hace continuas a las funciones suma y producto por escalares.

Kolmogorov caracterizó a los e.v.t. Un año posterior, en 1935, J. von Neumann desarrolla la teoría de los e.v.t., introduce e investiga a los destacados espacios localmente convexos (e.l.c.), idea que generaliza a la noción de espacio normado. Para una presentación más detallada de estos espacios ver la publicación de Treves-Figueiredo [15] donde el lector encontrará la teoría de los e.v.t. y sobre la teoría de distribuciones. Durante las décadas de los años 1940's-50's el grupo francés Bourbaki realizó una investigación de los e.v.t. (1953) contribuyendo hacia teorías modernas en el campo del análisis.

8. M. Stone. Operadores sobre Espacios de Hilbert. Alrededor de los años 1920's y 30's la matemática estuvo fuertemente en actividad gracias al liderazgo de D. Hilbert y su Escuela; Gotinga era el centro de gran agitación científica. En particular se va desarrollando el análisis moderno gracias a la presencia de notables analistas como F. Riesz, von Neumann, entre otros, y en Polonia la extraordinaria labor de $\mathrm{S}$. Banach y sus discípulos. Debemos remarcar que parte de este escenario se debió a que en forma paralela la física teórica trataba de explicar cuestiones en el mundo del átomo. La tendencia de los matemáticos era establecer puentes entre la física y la matemática; recordemos que el clásico problema de Dirichlet surgió en la teoría del potencial la que tuvo mucha influencia en las investigaciones teóricas en el cálculo de variaciones, teoría que condujo a Fredholm a la teoría de las ecuaciones integrales y ésta al análisis funcional. Así, en tales circunstancias se investigaron los operadores acotados y no acotados sobre espacios de dimensión infinita por parte de analistas de la época, entre los que estuvo el matemático norteamericano M. Stone.

8.1. El Libro de Stone. Las primeras contribuciones de Stone están en el período 1929-30, y están concentrados en los espacios de Hilbert y en el estudio organizado de operadores actuando sobre tales espacios; también aporta a la teoría espectral de transformaciones sobre espacios de Hilbert. El libro de Stone, citado en la sección 6, fue escrito en 1932, fue una excelente contribución a la naciente teoría del análisis funcional; surgía un nuevo análisis matemático con énfasis en los espacios de Hilbert y de los operadores actuando sobre ellos. Mucho de lo contenido en el libro fueron sus propias investigaciones. Es una obra con valor histórico pues fue escrito cuando la teoría estaba en pleno crecimiento y la información estaba en revistas. El libro es voluminoso y tiene diez capítulos que pasamos muy brevemente a comentar.

Stone da la noción de espacio, luego la de espacio de Hilbert abstracto $H$. Define: $H$ es completo si $f_{n}$ en $H$ satisface $\left\|f_{m}-f_{n}\right\| \rightarrow 0, m, n \rightarrow \infty$, entonces existe $f \in H$ tal que $\left\|f_{n}-f\right\| \rightarrow 0, n \rightarrow \infty$. Relaciona a los espacios $H$ con la teoría de la medida de Lebesgue; estudia al espacio $L^{2}$, que es un espacio de Hilbert. En el segundo capítulo estudia a los operadores en espacios de Hilbert $H(T f=g, f, g \in H)$; el adjunto $T^{*}$ de $T$ es definido vía $(T f, g)=\left(f, T^{*} g\right)$; $T$ es autoadjunto si $T=T^{*}$. $T$ es un operador lineal acotado si $\|T f\| \leq c\|f\|, c$ constante; $T$ es un operador isométrico si $(T f, T g)=(f, g)$... Stone introduce otras definiciones de operadores y de sus propiedades, tal como actualmente se estudian en un curso de análisis funcional. Luego Stone dá ejemplos de operadores resaltando la importancia de los espacios de Hilbert abstractas (estudia las matrices infinitas). Un ejemplo es dedicado a los operadores integrales definidos sobre $L^{2}$. También estudia operadores diferenciales ordinarias sobre $L^{2}$.

En seguida Stone nos conduce a la idea de espectro (en sus diferentes contextos) y a la de resolvente de un operador; considera el problema: "estudiar la ecuación $T f-\alpha f=g$, donde $\alpha$ es un número complejo dado, $g \in H$ también dada”. En relación a este problema dá la definición: Sea $T$ un operador lineal, $T_{\alpha}=T-\alpha I, T_{\alpha}^{-1}$ cuando exista. El espectro puntual de $T$ es el conjunto de puntos $A(T)$ en el $\alpha$-plano complejo donde $T_{\alpha}$ no tiene inverso. El espectro continuo de $T$ es el conjunto de puntos $B(T)$ donde $T_{\alpha}^{-1}$ existe y es un operador lineal no acotado cuyo dominio es denso en todas partes en $H$. El espectro residual de $T$ es el conjunto de puntos $C(T)$ tal que $T_{\alpha}^{-1}$ existe y es un operador lineal con dominio no denso en todas partes en $H$. El conjunto resolvente de $T$ es el conjunto de puntos $D(T)$ tal que $T_{\alpha}^{-1}$ existe y es un operador lineal acotado con dominio denso en todas partes en $H$. El espectro de $T$ es el conjunto de puntos $S(T)=A(T) \cup B(T) \cup C(T)$.

Stone prueba que los conjuntos $A, B, C$ y $D$ son exclusivos mutuamente. Debemos observar que actualmente se define el espectro de un operador como el complemento, respecto al plano complejo, del conjunto resolvente. Así mismo, sufrieron algunas modificaciones otras definiciones.

En el quinto capítulo Stone presenta sus propias investigaciones sobre los operadores auto-adjuntos basado en métodos de Stieltjes; usa la integral (de Stieltjes) $\int_{-\infty}^{\infty} F(\lambda) d \rho(\lambda)$, donde $F(\lambda), \quad-\infty<\lambda<\infty$, 
es una función de valor complejo, continua y acotada; $\rho(\lambda)$ es una función de variación acotada. Por otro lado, por conveniencia se redefine el espectro de un operador $T$ sobre un espacio de Hilbert siendo el conjunto $\sigma(T)=\{\lambda \in \mathbb{C} / T-\lambda I$ no es invertible $\}$. Si $\lambda \in \sigma(T)$, se tiene $|\lambda| \leq\|T\|$, es decir, $\sigma(T)$ es un conjunto acotado. Por otro lado, el conjunto $\rho(T)=\left\{\lambda \in \mathbb{C} /(T-\lambda I)^{-1}\right.$ existe $\}$ se llama el conjunto resolvente; $R_{\lambda}=(T-\lambda I)^{-1}$ es el operador resolvente. En el siguiente capítulo Stone desarrolla el cálculo de operadores general aplicado a los operadores auto-adjuntos, lo que es hecho en base a la integral de Radon-Stieltjes. Con los recursos obtenidos con su cálculo, Stone ataca el problema: “determinar bajo que condiciones podemos conectar dos operadores autoadjuntos $T_{1}$ y $T_{2}$ vía la relación $T_{2}=U T_{1} U^{-1}$, donde $U$ es un operador unitario". Para el caso de operadores limitados el problema fue resuelto por Hellinger en 1909 y por Hahn en 1912. Estas investigaciones condujeron a una completa caracterización de los operadores auto-adjuntos.

En el octavo capítulo Stone estudia los operadores unitarios y normales basados en la teoría de los operadores auto-adjuntos. $T$ es normal si $T T^{*}=T^{*} T$ y son una generalización de los operadores autoadjuntos que satisfacen $\left(T^{*} T\right)^{*}=T^{*} T$. Se dá la factorización: "Sea $T$ un operador lineal acotado, cuyo dominio es el espacio de Hilbert de una manera injectiva sobre si mismo. Entonces T puede ser factorizado en una de las dos formas $T=T_{1} U_{1} o ́ T=U_{2} T_{2}$, donde $T_{1}$ y $T_{2}$ son dos operadores auto-adjuntos, acotados y definidos positivos; $U_{1}$ y $U_{2}$ son dos operadores unitarios". En el próximo capítulo Stone estudia los operadores simétricos $((T x, y)=(x, T y), \forall x, y$ en el dominio de $T)$. Investiga sucesiones de operadores simétricos. Finalmente todo lo expuesto en los capítulos previos Stone los aplica a temas que investigaban entonces, como fueron los operadores integrales, los operadores diferenciales ordinarios de primer y segundo orden.

Observación 1. El libro de M. Stone, [14], es de gran valor matemático e histórico dado que fue escrito en una etapa en que la teoría de los espacios de Hilbert y de los operadores actuando sobre tales espacios, era aún una teoría en pleno desarrollo. Stone tuvo un gran sentimiento por los aspectos didácticos y por la enseñanza de la matemática. Consideramos que aún cuando actualmente disponemos de libros sobre análisis funcional, el libro citado conservará su valor matemático y de utilidad a posteriores generaciones.

9. Axiomatización de los Espacios de Hilbert. John von Neumann. Bajo la dirección de Hilbert, J. von Neumann trabajó en la axiomatización de los espacios de Hilbert durante los años 1920's y 30's. La Escuela Polaca y la de Gotinga eran los centros donde se producían grandes progresos; era la época en que las ideas topológicas, basadas en una sólida aplicación de la teoría de conjuntos y de la medida e integral de Lebesgue, contribuyeron a la formalización de nuevas teorías más generales; así surgieron los espacios normados y una teoría de operadores sobre tales espacios. En el período 1910-1935 las condiciones estuvieron dadas para la solución de buen número de problemas del análisis funcional, progreso que debemos fundamentalmente a F. Riesz, Helly, Hilbert, Schmidt, Banach, Fréchet, entre otros. En este escenario remarcamos la obra de S. Banach, cuyo libro hemos comentado anteriormente (ver sección 6), y por su interés analítico-histórico mencionemos los siguientes fundamentales resultados:

(i) (Banach - Steinhaus). "Sea $X$ un espacio de Banach, $Y$ un espacio vectorial normado y $F$ la familia de todas las funciones lineales acotadas de $X$ en $Y$. Si para todo $x \in X$, el conjunto $\{\|T x\| / T \in F\}$ es acotado, entonces el conjunto $\{\|T\| / T \in F\}$ es acotado". Este resultado fue probado y publicado en 1927.

(ii) (T. del Gráfico Cerrado), "Sean $X$ e $Y$ dos espacios de Banach y $T: X \rightarrow Y$ un operador lineal. Entonces, $T$ es acotado si y solo si el gráfico de T es cerrado en $X \times Y$ ".

(iii) (T. de la Aplicación Abierta) "Sean $X$ e $Y$ dos espacios de Banach, $T: X \rightarrow Y$ un operador lineal sobre. Entonces, para cualquier $U \subset X$ abierto, el conjunto $T(U)$ es un conjunto abierto en $Y$ ”.

Banach, en 1922, publicó el llamado teorema del punto fijo. Así, con el aporte de la Escuela Polaca, básicamente surgió el análisis funcional. Pero, ¿qué sucedía, también, en Gotinga?...

9.1. J. von Neumann, A. F. y Mecánica Cuántica. En Gotinga David Hilbert y sus discípulos contribuían con el desarrollo del análisis moderno, trataban de explicar dificultades que habían surgido en la naciente física cuántica. El ambiente de gran actividad científica estimuló a Hilbert y a sus alumnos el estudio de la teoría espectral; así, en 1926 Pascual Jordan y Paul Dirac elaboraron, en forma idependiente, la teoría de transformación con el objetivo de unificar las teorías sobre la mecánica cuántica de Heisenberg y Schrödinger. Recordemos que Dirac introdujo en este ocasión la conocida distribución $\delta$, en una época 
que ella no era aceptada por todos los matemáticos. En este año, 1926, un joven matemático húngaro llega a Gotinga para trabajar con Hilbert como su ayudante. Su nombre era: John von Neumann.

En esa época ya se conocía que los espacios $\ell^{2}$ y $L^{2}$ eran isomorfos e isométricos y eran los únicos espacios de Hilbert entonces conocidos; ahí aparecen las condiciones de normalización $\sum_{n=1}^{\infty}\left|x_{n}\right|^{2}=1$, en la mecánica matricial, y la condición $\int|f(x)|^{2} d x=1$, en la mecánica ondulatoria, y von Neumann dedujo que las teorías de Heisenberg y de Schrödinger eran equivalentes y así el reto fue construir una teoría general en donde $\ell^{2}$ y $L^{2}$ fueran casos particulares. ¡Ya estaban gestándose los nuevos espacios de Hilbert abstractos!. En el período 1929-1932 von Neumann escribió tres notables trabajos en donde desarrolla su teoría axiomática de estos nuevos espacios; introduce también una teoría de operadores normales y hermitianos. Mencionemos que por estos años se investigaban algunos espacios de funciones, así como los operadores actuando sobre tales espacios. En su libro sobre los fundamentos matemáticos de la mecánica cuántica von Neumann dice: "el objetivo de este libro es presentar la mecánica cuántica en una presentación unificada la cual, tan útil como sea posible, es matemáticamente rigurosa”. En su obra, parte con un espacio vectorial $H$, sobre los complejos, define al producto interno, $(f, g)$, vía los axiomas:

(i) $(\alpha f, g)=\alpha(f, g)$,

(ii) $\left(f_{1}+f_{2}, g\right)=\left(f_{1}, g\right)+\left(f_{2}, g\right)$,

(iii) $(f, g)=\overline{(g, f)}$,

(iv) $(f, f) \geq 0$,

(v) $(f, f)=0$ si y solo si $f=0$.

Luego define: $f$ y $g$ son ortogonales si $(f, g)=0$ y escribe $f \perp g ;\|f\|=(f, f)^{\frac{1}{2}}$ es la norma de $f$ y $d(f, g)=\|f-g\|$ es una métrica en el espacio $H$.

El primer teorema que von Neumann prueba en su libro es: “ $|(f, g)| \leq\|f\|\|g\|, \forall f, g \in H$. Se tiene la igualdad si f y g son idénticos salvo por un factor complejo constante”. Luego prueba las propiedades fundamentales de la norma:

(i) $\|f\| \geq 0 ;\|f\|=0$ si y solo si $f=0$,

(ii) $\|a f\|=|a|\|f\|, a$ complejo,

(iii) $\|f+g\| \leq\|f\|+\|g\|$.

Por otro lado, si $\left\{\phi_{n}\right\}, n=1,2,3, \ldots$ es un conjunto ortogonal, entonces von Neumann prueba que la serie $\sum_{n}\left(f, \phi_{n}\right) \overline{\left(g, \phi_{n}\right)}$ es absolutamente convergente; si $f=g$ se obtiene la desigualdad de Parseval $\sum_{n} \mid\left(f,\left.\phi_{n}\right|^{2} \leq\|f\|^{2}\right.$. Dá atención a los operadores actuando sobre los espacios de Hilbert, así un operador lineal $R$ es hermítico sobre un espacio de Hilbert $H$ si $(R f, g)=(f, R g), \forall f, g \in H$. También considera al operador proyección y verifica: "Sea $M$ un subespacio cerrado de $H$; si $f \in H$ entonces $f=g+h$ de un modo único donde $g \in M, h \in(H-M)$. El operador proyección $P_{M}$, asociado a $M$, es definido vía $P_{M}(f)=g$ ”. Remarcamos que si $Y$ y $Z$ son dos subespacios cerrados de $H$, por definición:

$$
Y-Z=\{x \in Y /(x, y)=0, \forall y \in Z\}
$$

Estos, y otros, resultados fueron publicados por von Neumann en 1929; en otro artículo introduce las topologías fuerte y débil en un espacio de Hilbert. Verifica que un operador lineal $R$ es acotado si $\exists$ una constante $M>0$ tal que $\forall f \in H$ se tiene $\|R f\| \leq M\|f\|$; define $\|R\|$ al ínfimo de tales $M$ 's. Verifica que $R$ es acotado si y solo si $R$ es continua.

Von Neumann contribuyó mucho con la construcción del análisis funcional. Probó que si un operador lineal es continuo en un punto, lo será también en todo punto del dominio, y luego será acotado. Define al operador adjunto $R^{*}$; y $R$ es operador normal si $R R^{*}=R^{*} R$ y es llamado unitario si $R R^{*}=R^{*} R=I$, donde $I$ es el operador identidad; en este caso se tiene que $R^{*}=R^{-1}$ y que $\|R f\|=\|f\|$. Von Neumann investiga el problema de los valores propios y así llega a la teoría espectral de operadores, teoría que fue muy útil en la mecánica cuántica. El mencionado libro tiene 6 capítulos; en el segundo von Neumann desarrolla la teoría de los espacios de Hilbert abstractos y tiene 11 secciones. Esta teoría es el lenguaje matemático que es fundamental para penetrar al misterioso mundo cuántico.

10. Panorama de Otros Dominios del Análisis Funcional. Como ya sabemos a fines del siglo XIX e inicios del siglo XX fueron gestándose espacios donde los elementos ya no eran necesariamente números reales o complejos; la idea de punto fue generalizado, podía ser de naturaleza arbitraria, una función, una 
matriz, etc. Así surgieron los espacios de funciones, los cuales fueron dotados de una estructura vectorial, algebraica y topológica. Surgieron dos espacios fundamentales: los espacios de Hilbert y los de Banach. Estamos alrededor del año 1930. En 1932 se publicaron tres famosos libros, que ya hemos comentado, y que motivaron nuevas investigaciones en el análisis funcional y aplicaciones. Comentemos algunas de ellas.

10.1. Espacios Vectoriales Topológicos.. Por aquellos años, 1930's, el análisis funcional sigue progresando, se crean nuevas ramas en donde se inter-relacionan métodos algebraicos con los topológicos. von Neumann contribuye con la teoría espectral y con ciertas subálgebras involutivas que habrían de conducir a las álgebras de von Neumann; por otro lado y en esta dirección, el matemático ruso I. Gelfand funda, en 1941, la teoría general de las álgebras normadas, extendiendo la teoría espectral de F. Riesz a álgebras normadas arbitrarias. También introdujo la fórmula del radio espectral; en colaboración con M. Naimark introdujo las $C^{*}$-álgebras, un tema que investigó fuertemente en los años 1940's.

En la sección 7 vimos que el matemático ruso A. Kolmogorov introdujo los espacios vectoriales topológicos (e.v.t) como culminación de todo un proceso de investigaciones, lo que fue llevado a la condición de teoría por J. von Neumann. Antes de los e.v.t.'s las nociones eran métricas, ahora son topológicas; por ejemplo, $A$ es un conjunto acotado en un e.v.t. $X$ si para toda sucesión $\left\{x_{n}\right\}$ en $A$ y toda sucesión de escalares convergiendo a cero, la sucesión $\left\{\lambda_{n} x_{n}\right\}$ converge a cero. Remarcamos que la noción de convergencia también es dada topológicamente. En 1935 J. von Neumann introduce e investiga los espacios localmente convexos (e.l.c.) motivado por la investigación de los conjuntos convexos; estos espacios son una generalización adecuada a la noción de espacio normado. Un e.l.c. es un espacio vectorial $E$ provisto de una topología definida por una base de filtros de vecindades del origen, una familia de conjuntos convexos, absorventes y equilibrados. (Para los detalles ver, por ejemplo, Treves-Figueiredo [15], pag. 17).

De esta manera, en el análisis matemático moderno se tuvo la teoría de los e.v.t. cuyo estudio organizado, en 1953, debemos al grupo francés Bourbaki. Así en las décadas de los años 1940's y 1950's la matemática, a través del análisis, va a progresar de un modo muy estimulante, tanto en el campo teórico como en las aplicaciones.

10.2. La Teoría de Distribuciones.. En su libro "Linear Partial Differential Operators", 1963, el matemático Lars Hörmander dijo: "La historia de la teoría de distribuciones está intimamente conectada con la teoría de las ecuaciones en derivadas parciales. El estudio del problema de Cauchy condujo a ciertas distribuciones... Es en la forma final de Schwartz, donde la transformada de Fourier es una parte escencial, es que la teoría de distribuciones vino a ser un instrumento conveniente en las investigaciones de las ecuaciones en derivadas parciales".

Como sabemos, por los años 1920's, el físico británico P. Dirac introdujo la llamada "función” delta $\delta$ definida vía: $\delta(x)=0, \forall x \neq 0, \delta(0)=+\infty, \int_{-\infty}^{\infty} \delta(x) d x=1$, definición que no resiste a la teoría de Lebesgue sin embargo Dirac la usó con éxito para explicar las dificultades que había en la mecánica cuántica. Se verificó que si $f$ es una función continua sobre $\mathbb{R}$, entonces $f(a)=\int_{-\infty}^{\infty} f(x) \delta(a-x) d x, \forall a \in$ $\mathbb{R}$. Aún más, $\delta$ es infinitamente diferenciables, y si $f \in C^{k}(\mathbb{R})$ entonces se tiene

$$
f^{(k)}(a)=\int_{-\infty}^{\infty} f(x) \delta^{(k)}(a-x) d x, \forall a \in \mathbb{R} .
$$

Por otro lado, Heaviside introdujo la función (motivado por las aplicaciones):

$$
Y(x)=\left\{\begin{array}{cc}
0 & , x<0 \\
1 & , x>0
\end{array},\right.
$$

y se probó que $Y^{\prime}(x)=\delta(x)$; esto es, una no función es la derivada de una función!! Aún más,

$$
\frac{d}{d x} \log x=\frac{1}{x}-i \pi \delta(x)
$$

una ecuación que aparece en la mecánica cuántica.

La introducción y uso de la $\delta$ creó un clima de inquietud entre los matemáticos y clarificar este panorama llevó un promedio de 20 a 30 años y fue la Escuela Francesa la que renovó, modernizó y rigurizó el análisis; surgió el grupo N. Bourbaki para realizar tal tarea. En este ambiente el matemático francés Laurent 
Schwartz considera al espacio $C_{\circ}^{\infty}\left(\mathbb{R}^{n}\right)$ de las funciones infinitamente diferenciables con soporte compacto. En otras palabras, $D \equiv C_{\circ}^{\infty}\left(\mathbb{R}^{n}\right)$ es el espacio vectorial de todas las funciones $\phi$ de valor real o complejo, definidas sobre $\mathbb{R}^{n}$, las cuales tienen derivadas parciales continuas de todos los órdenes y tales que sus soportes están en un conjunto compacto contenido en $\mathbb{R}^{n}$. Si $\alpha=\left(\alpha_{1}, \alpha_{2}, \ldots, \alpha_{n}\right)$, siendo $\alpha_{i}$ un entero positivo, pongamos $|\alpha|=\alpha_{1}+\alpha_{2}+\ldots+\alpha_{n} \mathrm{y}$

$$
\partial^{\alpha} \equiv \frac{\partial^{|\alpha|}}{\partial x_{1}^{\alpha_{1}} \cdots \partial_{n}^{\alpha_{n}}} .
$$

Definición 2. $T: D \rightarrow \mathbb{R}(o \mathbb{C})$ es una distribución si $T$ es una funcional lineal y si para todo compacto $K \subset \mathbb{R}^{n}$ existe una constante $M_{k}>0$ y un entero $m \geq 0$ tal que

$$
|T(\phi)| \leq M_{k} \operatorname{máx}_{|\alpha| \leq m} \operatorname{máx}_{x \in \mathbb{R}^{n}}\left|\partial^{\alpha} \phi(x)\right|,
$$

para todo $\phi \in D$ talque $\operatorname{supp} \phi \subset K$.

Es usual usar la notación $T(\phi) \equiv<T, \phi>$.

Si $f \in L_{\text {loc }}^{1}\left(\mathbb{R}^{n}\right)$, definimos $T$ vía $<T, \phi>=\int f(x) \phi(x) d x, \forall \phi \in D$, entonces $T$ es una distribución; también, si la $\delta$ de Dirac se define vía $\langle\delta, \phi\rangle=\phi(0), \forall \phi \in D$, entonces $\delta$ es una distribución!! Para mayores detalles sobre la teoría de distribuciones ver [15], pag. 105.

10.3. Operadores Diferenciales Parciales.. En los años 1950's hubieron grandes progresos en el campo de las ecuaciones en derivadas parciales gracias a las aplicaciones del análisis funcional y de la teoría de distribuciones; se introdujeron nuevos espacios de funciones y se investigaron los operadores diferenciales parciales; en particular se estudió los operadores con coeficientes constantes en el problema de la existencia de una solución fundamental y en esta dirección un primer resultado general fue obtenido en 1954 por L. Ehrenpreis y por B. Malgrange en el periódo 1955-56. Un aporte fundamental fue dado por el matemático sueco Lars Hörmander quien publicó, en 1955 su tesis doctoral: "On the theory of general partial differential operators", trabajo que motivó muchas otras investigaciones, incluyendo las del propio Hörmander.

Por otro lado, L. Schwartz realizó un estudio de la transformada de Fourier desde un punto de vista de las distribuciones y ello permitió construir una nueva visión de las ecuaciones en derivadas parciales (EDP) y en este camino los e.v.t., en particular los e.l.c., fueron útiles en tal progreso. Dieudonné-Schwartz, en 1949, estudiaron resolver la ecuación $P u=f(*)$, la cual es una EDP linear con coeficientes constantes, $f$ es una función dada de valor complejo, definida sobre un abierto $D \subset \mathbb{R}^{n} ; f$ es infinitamente diferenciable. El objetivo fue saber si existe una solución $u$ de $(*)$ y si ella es también infinitamente diferenciable en $D$. Así mismo, la noción de solución fundamental fue muy importante pues conociendo tal solución se puede resolver la EDP: $P(S)=T(* *)$, donde $S$ es la distribución incógnita y $T$ es una distribución con soporte compacto dada. Así: "una distribución E se llama una solución fundamental del operador diferencial parcial $P$ si $P(E)=\delta$, donde $\delta$ es la medida de Dirac en el origen”. Entonces se tiene que $S=E * T$ (convolución) es solución de la ecuación $(* *)$ dado que $P(E * T)=(P(E) * T)=\delta * T=T$.

Según Horváth, uno de los más importantes éxitos de la teoría de distribuciones fue logrado en forma independiente por Malgrange y Ehrenpreis en 1952 al probar: "toda EDP lineal, con coeficientes constantes (no identicamente cero) tiene una solución fundamental”.

\section{Observación 2.}

1. En el período 1958-1971 N. Dunford - J. Schwartz escribieron un voluminoso tratado sobre operadores lineales en tres grandes volúmenes. Es una gran fuente de consulta.

2. Temas más actualizados, hasta 1971, pueden ser encontrados en "Topics in Operator Theory" de Richard Beals (University of Chicago Press).

3. A partir de los años 1950's los profesores A. Calderón - A. Zygmund desarrollaron la teoría de los operadores integrales singulares, teoría que tuvo fuertes conexiones con los operadores diferenciales parciales. Esto es otra hermosa historia!

4. Para otras referencias históricas del análisis funcional consultar [2] y [12].

ORCID and License

Alejandro Ortiz Fernández https: / / orcid.org/0000-0002-9380-4301

This work is licensed under the Creative Commons - Attribution 4.0 International (CC BY 4.0) 


\section{Referencias}

[1] Banach S. Théorie des Opérations Linéaires. Warszawa. 1932

[2] Bombal F. Los Orígenes del Análisis Funcional. Real Acad. de Ciencias de Madrid. 1994.

[3] Dieudonné J. History of Functional Analysis. North-Holland Elsevier Sc. 1983.

[4] Figueiredo D. Teoría Classica do Potencial. U. N. Brasilia. 1963.

[5] Figueiredo D. Introdução ãs Equações Integrais. Escola de Análise. São Paulo. 1977.

[6] Gelfand IM. Análisis Funcional. Dentro de la Matemática: su contenido, métodos y significado. Alianza Editorial. Vol. 3. Madrid. 1973.

[7] Kline M. El Pensamiento Matemático de la Antigüedad a nuestros Días. Alianza Editorial. Madrid. 2012.

[8] Ortíz A. Tópicos sobre Ecuaciones en Derivadas Parciales. Internet. PUCP. 2004.

[9] Ortíz A. La Matemática a Través de Clásicas Áreas. Vol. 3. Internet. PUCP. 2017.

[10] Ortíz A. Some Ideas on the Genesis of the Infinitesimal Calculus. Selecciones Matemáticas. 2021; 8(1):173-195.

[11] Ortíz, A. Some Ideas on the Evolution of Real Mathematical Analysis. Selecciones Matemáticas. 2021; 8(1):196-217.

[12] Pietsch A. History of Banach Spaces and Linear Operators. Birkhäuser. Boston. 2007.

[13] Riesz F, Nagy B. Functional Analysis. Frederick Ungar. Pub. 1955.

[14] Stone M. Linear Transformations in Hilbert Space and their Applications to Analysis. Am. Math. Soc. N. York. 1932.

[15] Treves F, Figueiredo D. Espaços Vetoriais Topológicos e Distribuições. Notas de Mat. 41IMPA. Brasil. 1968. 